\title{
Dealing with perioperative antiplatelet treatment for transurethral resection of the bladder: primum non nocere
}

\author{
François Audenet ${ }^{1}$, John P. Sfakianos ${ }^{2}$ \\ ${ }^{1}$ Department of Urology, Hôpital Européen Georges Pompidou, Université Paris Descartes, Paris, France; ${ }^{2}$ Department of Urology, Icahn School of \\ Medicine at Mount Sinai, New York, NY, USA \\ Correspondence to: François Audenet. Department of Urology, Hôpital Européen Georges Pompidou, Université Paris Descartes, Paris, France. \\ Email: francois.audenet@gmail.com. \\ Provenance: This is a Guest Editorial commissioned by Section Editor Xiao Li (Department of Urology, Jiangsu Cancer Hospital \& Jiangsu Institute \\ of Cancer Research \& Nanjing Medical University Affiliated Cancer Hospital, Nanjing, China). \\ Comment on: Prader R, De Broca B, Chevallier D, et al. Outcome of Transurethral Resection of Bladder Tumor: Does Antiplatelet Therapy Really \\ Matter? Analysis of a Retrospective Series. J Endourol 2017;31:1284-8.
}

Submitted Mar 22, 2018. Accepted for publication Mar 26, 2018.

doi: $10.21037 /$ tau.2018.03.24

View this article at: http://dx.doi.org/10.21037/tau.2018.03.24

The number of patients receiving antithrombotic treatment is increasing (1), thus increasing the number of patients under anticoagulant or antiplatelet treatment scheduled to undergo endo-urology surgery. Perioperative management of antithrombotic treatment is a matter of debate, based on the trade-off between the risk of cardiovascular event and the risk of perioperative bleeding. There are no formal guidelines and only few studies have addressed this issue, particularly for transurethral resection of the bladder (TURBT). The study recently published by Prader et al. in fournal of Endourology (2) is a non-inferiority monocentric retrospective study evaluating the mean length of stay between 2 groups of patients treated with TURBT: patients receiving antiplatelet therapy before surgery and patients naïve of treatment. Overall, 117 consecutive patients were included in each arm. Patients receiving anticoagulant were excluded. As expected, patients receiving antiplatelet treatment were significantly older (79 vs. 66 years), with significantly more comorbidity (hypertension, coronary artery disease, atrial fibrillation, diabetes, dyslipidemia, stroke). This population was more frequently operated on under regional anesthesia (43\% vs. 27\%). Interestingly, patients under antiplatelet had significantly smaller and lower stage bladder tumors, which could be explained by earlier diagnosis in relation with hematuria. In the antiplatelet group, only $18 \%$ of the patients had treatment cessation before surgery, the other being withheld or relayed by low-dose aspirin. Overall, $80 \%$ of the patients were operated on under aspirin $75 \mathrm{mg}$. The study showed that the mean length of stay after TURBT in patients receiving antiplatelet therapy before surgery was significantly longer compared to patients naïve of treatment (2.9 vs. 2.3 days, $\mathrm{P}=0.024$ ). However, the difference didn't reach the predefined 1-day difference to make it clinically relevant. Furthermore, subgroup analysis demonstrated that aspirin didn't lead to a significant difference in the length of stay, whereas clopidogrel was associated with longer stay in hospital. Although the numbers were limited, patients receiving clopidogrel $(\mathrm{N}=32)$ were also at higher risk of transfusion (13\%), failure of Folley catheter removal (22\%), reintervention for hemostasis (13\%) and cardiovascular/ neurovascular event (3.1\%).

During TURBT, significant intraoperative or immediate postoperative bleeding occurs in $2-13 \%$ of the patients in the literature and it is the most common complication (3). Antithrombotic treatment is a major risk factor. However, the risk of thromboembolic complications in case of cessation could exceed the risk of bleeding associated with the procedure. In order to compare the effect of maintenance or interruption of aspirin before surgery, the STRATAGEM (Strategy for Managing Anti-platelet Therapy in the Perioperative Period of Non Coronary Surgery) trial compared the cumulative rates of major thrombotic and bleeding events at 30 days postoperatively 
in patients randomized to aspirin $75 \mathrm{mg}$ daily $(\mathrm{N}=145)$ or placebo $(\mathrm{N}=146)$ (4). Patients were receiving antiplatelet therapy for secondary prevention of coronary artery disease, stroke, transient ischemic attack or peripheral vascular ischemic disease and undergoing elective intermediate or high-risk elective non-cardiac surgery, including $15.5 \%$ of urologic procedures. While the study was underpowered, there was no significant difference in major thrombotic or bleeding events between preoperative maintenance or interruption. Major adverse events occurred in 31 patients $(10.7 \%)$, including 6 thromboembolic events in the aspirin group and 5 in the placebo group, 10 bleeding events in the aspirin group and 10 in the placebo group.

In the study published by Prader et al. the results were concordant with only 2 thrombotic events and 4 hemorrhagic events requiring reintervention in the antiplatelet group, of whom $80 \%$ had TURBT under lowdose aspirin. Other studies also found that continued use of antiplatelet therapy was not associated with an increased risk of bleeding or reintervention (5). In a recent review of the literature, Naspro et al. even suggested that grade and stage may have a greater influence on transfusion requirements than concomitant antithrombotic treatment (6). Furthermore, it has even been demonstrated that the highest cardiovascular risk occurs immediately after abrupt interruption of antiplatelet therapy, causing platelet rebound, inflammatory and prothrombotic state, which promotes platelet aggregation (7). Consequently, in most cases, low-dose aspirin can be continued perioperatively without a significantly increased risk of major bleeding. However, clopidogrel is a much more powerful antiplatelet therapy and relay with aspirin 5 days before TURBT is usually the rule to prevent bleeding complications. As pointed by the authors, due to the hepatic transformation into active form, there is an important variability in the metabolism that could explain a higher risk of bleeding is some patients bridged with aspirin. While highlevel of evidence is missing, clinical guidance should be based on a risk-adapted strategy, involving urologists, anesthesiologists and cardiologists. Most of the currently available literature support continuation of aspirin in patients undergoing TURBT. More evidence should be generated to evaluate the optimal duration of clopidogrel withdrawal.

\section{Acknowledgements}

None.

\section{Footnote}

Conflicts of Interest: The authors have no conflicts of interest to declare.

\section{References}

1. Apenteng PN, Gao H, Hobbs FR, et al. Temporal trends in antithrombotic treatment of real-world UK patients with newly diagnosed atrial fibrillation: findings from the GARFIELD-AF registry. BMJ Open 2018;8:e018905.

2. Prader R, De Broca B, Chevallier D, et al. Outcome of Transurethral Resection of Bladder Tumor: Does Antiplatelet Therapy Really Matter? Analysis of a Retrospective Series. J Endourol 2017;31:1284-8.

3. Collado A, Chéchile GE, Salvador J, et al. Early complications of endoscopic treatment for superficial bladder tumors. J Urol 2000;164:1529-32.

4. Mantz J, Samama CM, Tubach F, et al. Impact of preoperative maintenance or interruption of aspirin on thrombotic and bleeding events after elective noncardiac surgery: the multicentre, randomized, blinded, placebo-controlled, STRATAGEM trial. Br J Anaesth 2011;107:899-910.

5. Picozzi S, Marenghi C, Ricci C, et al. Risks and complications of transurethral resection of bladder tumor among patients taking antiplatelet agents for cardiovascular disease. Surg Endosc 2014;28:116-21.

6. Naspro R, Lerner LB, Rossini R, et al. Perioperative antithrombotic therapy in patients undergoing endoscopic urologic surgery: where do we stand with current literature? Minerva Urol Nefrol 2018;70:126-136.

7. Ho PM, Peterson ED, Wang L, et al. Incidence of death and acute myocardial infarction associated with stopping clopidogrel after acute coronary syndrome. JAMA 2008;299:532-9. Erratum in: JAMA 2008;299:2390.

Cite this article as: Audenet F, Sfakianos JP. Dealing with perioperative antiplatelet treatment for transurethral resection of the bladder: primum non nocere. Transl Androl Urol 2018;7(Suppl 2):S258-S259. doi: 10.21037/tau.2018.03.24 